\title{
A fraction to fraction study of photo-oxidation of BAL 150 crude oil asphaltenes
}

\author{
A. Boukir ${ }^{1,2}$, M. Guiliano ${ }^{1}$, L. Asia ${ }^{1}$, A. El Hallaoui ${ }^{2}$ and G. Mille ${ }^{1, *}$ \\ ${ }^{1}$ Laboratoire de Chimie Analytique de l'Environnement, Faculté des Sciences et Techniques de Saint-Jérôme, \\ Université d'Aix-Marseille, 13397 Marseille Cedex 20, France \\ ${ }^{2}$ Laboratoire de Chimie Organique, Faculté des Sciences Dhar Mehraz, BP. 1796 Atlas, Fes, Morocco
}

\begin{abstract}
Sun light plays an important role in the degradation of oil spilled at sea or ground surface. Nevertheless, the efficience of photo-oxidation as a weathering process was hardly studied in asphaltenes, the most recalcitrant of petrogenic contaminants. Asphaltenes precipitated from Blend Arabian Light crude oil topped at $150{ }^{\circ} \mathrm{C}$ were exposed to UV and visible light sources for time durations up to 140 days, analyzed globally, then fractionated by sequential elution solvent chromatography. FTIR spectroscopy, elemental analysis, NMR, UV-fluorescence, GC and GC/MS analyses were implemented to investigate structural changes potentially induced by photo-oxidation. No obvious effect could be detected by overall approaches, whereas fraction to fraction analyses evidenced significant variations in the gravimetric distribution of asphaltene constituents, accompanied by changes in functionality linked to limited oxidation processes. Under our experimental conditions, most observed changes were suggestive of an increase in carbonyl, phenolic, sulfoxyde and carboxylic groups after light exposure, without significant alteration of the hydrocarbon skeletons of polyaromatic and alkyl compounds associated with the asphaltene matrix.
\end{abstract}

Key words. Crude oil asphaltenes - chromatographic fractionation - photo-oxidation - FTIR - UV-fluorescence.

\section{Introduction}

Among several intricate physical, physico-chemical, and biological factors, natural light plays an important role [1-6] in the mitigation of oil spills at sea or ground surface. Nevertheless, little is known about the contribution of photooxidation to the lengthy degradation of asphaltenes [7-11], the most recalcitrant fraction to weathering processes. Their extremely complex composition probably accounts for this lack of data, by making gradual changes outstandingly difficult to investigate.

The chemical nature of asphaltenes is poorly defined. In fact, they correspond to a solubility class of constituents: these precipitated from crude oils by adding at least 40 volumes of normal alkanes $\left(n \mathrm{C}_{5}\right.$ to $\left.n \mathrm{C}_{7}\right)$. Asphaltenes contain lamellar macromolecules resulting from the condensation of polyaromatic, naphthenic and heterocyclic nuclei, bearing alkyl side chains and sometimes functionalized. Their molecular weight varies from several hundreds to several thousands [12,13]. Cross linked by alkyl, sulphur and oxygen bridges, the polycyclic sheets constitute a matrix that traps various aliphatic hydrocarbons, and form micelles spread among the other petroleum constituents (hydrocarbons and resins).

Addressing chemical changes in such structures is a technical challenge, and hence separating samples into different fractions is an unescapable step before analysis. To investigate the effects of light in asphaltenes isolated from Blend Arabian Light crude oil, samples were fractionated by sequential elution solvent chromatography [14]. This technique, suitable for liquid coals [15-16], and adapted to crude oil asphaltenes [17] was selected, for it separates compounds according to functionalities. Subsequent analyses involved gravimetry, elemental analysis, NMR, UV-fluorescence and chiefly FTIR spectroscopy.

\section{Materials and methods}

The effects of light exposure were studied in asphaltenes separated from Blend Arabian Light topped at $150{ }^{\circ} \mathrm{C}$ (BAL 150). In addition, a number of asphaltenes from several other crude oils (Rospo Mare, Ekofisk, Oman, Handil, Irak, Saoudian (PBS), Kuwait, Shengli, Bach Ho, Zarzaïtine, Blend Arabian Medium and Bunker C) were fractionated and investigated to elucidate some FTIR data.

\section{Precipitation of asphaltene fractions}

Asphaltenes were precipitated in compliance with the NFT 60-115 AFNOR standard [18]. Crude oil (5 g) was mixed to n-heptane $(200 \mathrm{~mL})$ in a $250 \mathrm{~mL}$ round bottom flask, heated to reflux $\left(90{ }^{\circ} \mathrm{C}\right)$ under agitation during $2.5 \mathrm{hr}$, and then allowed to settle for $12 \mathrm{hr}$ at room temperature. This solution was filtered on a glass microfiber filter (Whatman GF/A, porosity $0.45 \mu \mathrm{m}$ ). Warm heptane was used to drain out the precipitate coating the flask wall, and to thoroughly rinse the solid collected on the filter. The solid was dissolved with dichloromethane; the solution was then concentrated in a rotary evaporator and dried under nitrogen flow up to complete solvent elimination. In the case of BAL 150 crude oil, this technique yielded an asphaltene fraction of about $3 \%$.

\section{Photo-oxidation}

Films of pulverized asphaltenes ( 0.2 to $0.5 \mathrm{~mm}$ thick) were exposed to light at room temperature. Samples were irradiated at room temperature for 30,80 and 140 days, by two

* Correspondence and reprints

Received May 26, 1998; revised August 28, 1998; accepted September 8, 1998. 
30 Watt fluorescent lamps covering the UV and visible ranges (L80/79R and $\mathrm{L} 58 \mathrm{~W} / 10$ Osram respectively), at an average $60 \mathrm{~cm}$ distance of the light sources, corresponding to an irradiation of 30 Einstein $\mathrm{m}^{-2}$ per day.

\section{Fractionation}

Silica gel (Merck $63-200 \mu \mathrm{m})$ was soxhlet extracted with a dichloromethane/methanol mixture (1:1) during $72 \mathrm{hr}$, then dried and activated by exposure at $120^{\circ} \mathrm{C}$ for $12 \mathrm{hr}$. A $31 \mathrm{~cm}$ long, $1 \mathrm{~cm}$ in diameter, open glass column, obturated at bottom by glass wool pad (washed as silica gel), was packed with $12 \mathrm{~g}$ of silica gel deactivated by addition of $5 \%$ of deionized water. The silica gel was soaked with hexane, and successively rinsed with $100 \mathrm{~mL}$ hexane, $100 \mathrm{~mL}$ chloroform and eventually $100 \mathrm{~mL}$ methanol in order to drain off all impurities. Dry asphaltenes $(200 \mathrm{mg}$ ) were laid onto the column and progressively eluted with: 1. hexane; 2. hexane/toluene $(85: 15) ; 3$. chloroform; 4. chloroform/ diethylether (90:10); 5. diethylether/ethanol (97:3); 6 . methanol; 7. ethanol/chloroform (3:97); 8. Ethanol/tetrahydrofuran (3:97); 9. Ethanol/pyridine (3:97); 10. pyridine; 11. triethylamine; 12 . acetone; and 13 . ethylacetate $(20 \mathrm{~mL}$ each) - a sequence in ascending order of dissolving power (increasing solvent strength $\left(\varepsilon^{\circ}\right)$ and Hildebrand parameters $[14,17]$ : dispersion, dipole-dipole, proton donor, proton acceptor).

\section{Summarily:}

a) Elution started with a series of solvents in which asphaltenes are either partially (1 and 2) or completely soluble (3 and 4), and with polar solvents in which they are slightly soluble only (5 and 6); this first series of solvents hence eluted asphaltenes of relatively low functionality (fractions 1 to 6 ).

b) After silica gel deactivation by methanol (6), the four subsequent elutions used solvents of increasing polarities and strengths, in which asphaltenes are completely soluble ; this second series of solvents hence eluted acid or basic asphaltenes of increasing functionality (fractions 7 to 10).

c) After pyridine elution, 91 to $95 \%$ of the asphaltene content has been collected; the last series of solvents (11 to 13) collected another 1 or $2 \%$ (eventually assembled, and referred to as fraction 11).

After elution, fractionated asphaltenes were concentrated in a rotary evaporator, dried under nitrogen, weighed and stored at low temperature $\left(5-8{ }^{\circ} \mathrm{C}\right)$ for latter analysis. Blanks were regularly performed to check that glassware, silica and solvents were free of any contamination.

\section{Spectral analysis}

FTIR spectra were obtained on a 20 SXB Nicolet spectrometer by co-addition of 128 interferograms at a $2 \mathrm{~cm}^{-1}$ resolution. Each fraction was dissolved in dichloromethane at a concentration ranging from 25 to $50 \mathrm{mg} \mathrm{L}^{-1}$; this solution $(25 \mu \mathrm{L})$ was laid onto a $\mathrm{KBr}$ plate and evaporated under a stream of nitrogen. Careful preparation of homogen films was essential to insure data reproducibility; every spectrum was checked to verify the absence of eluting solvents and/or dichloromethane IR bands.
A Bruker spectrometer was used to record ${ }^{1} \mathrm{H}$ NMR spectra at $200 \mathrm{MHz}$, and ${ }^{13} \mathrm{C}$ NMR spectra at $50 \mathrm{MHz}$, the latter by co-addition of 20000 to 30000 accumulations (relaxation time: $10 \mathrm{~s}$ ). Deuterated chloroform was used as solvent.

UV- fluorescence spectra between 250 and $600 \mathrm{~nm}$ were obtained on a 3000 Perkin-Elmer spectrometer, using the synchronous excitation emission technique $(\Delta \lambda=23 \mathrm{~nm})$ in a $1 \mathrm{~cm}$ cell. Tetrahydrofuran was used as solvent.

Gas chromatograms were obtained on a Vega 6000 Fisons chromatograph, with a CP SIL 5 CB $25 \mathrm{~m} \times 0.25 \mathrm{~mm} \times$ $0.25 \mu \mathrm{m}$ capillary column, with temperature controlled from 70 to $285{ }^{\circ} \mathrm{C}$ at $5{ }^{\circ} \mathrm{C} \mathrm{min}-1$, at $285{ }^{\circ} \mathrm{C}$ for $25 \mathrm{~min}$, from 285 to $295{ }^{\circ} \mathrm{C}$ at $10{ }^{\circ} \mathrm{C} \mathrm{min}{ }^{-1}$, and at $295{ }^{\circ} \mathrm{C}$ for $10 \mathrm{~min}$.

GC/MS analysis used a Hewlett Packard 5989 A mass spectrometer (electron energy $70 \mathrm{eV}$, source temperature $240{ }^{\circ} \mathrm{C}$ ) coupled with a HP 5890 chromatograph equipped with a splitless injector. Separations were obtained under the following conditions: $60 \mathrm{~m} \times 0.32 \mathrm{~mm} \times 0.25 \mu \mathrm{m}$ DB-5 MS $(\mathrm{J} \& \mathrm{~W})$ capillary column, temperature controlled as above.

\section{Results and discussion}

\section{Global analyses}

Table I presents the data obtained from elemental analysis of total asphaltenes, showing little difference before and after light exposure. Main bands detected in FTIR spectra are listed in table II and assigned to respective functions according to current knowledge [11,19,20]. As preliminary reported in a previous note [21], this global approach failed to evidence significant changes even after maximal light exposure (140 days). Specific structural indexes, computed from area ratios of characteristic IR bands, were equivalent, except the oxidation index and aliphaticity index, the latter exhibiting minor variations only. UV-fluorescence spectra before or after light exposure were not significantly different; similarly, ${ }^{1} \mathrm{H}$ NMR and ${ }^{13} \mathrm{C}$ NMR spectra were unchanged. With reference to current knowledge [17,22-30], these negative data suggested that hydrocarbon skeletons underwent little alteration if any during light exposure.

\section{Fraction to fraction analyses}

Table III presents the relative changes in gravimetric distribution of asphaltenes among the different eluted fractions. Before light exposure, the most abundant fractions were those extracted by hexane/toluene ( $11.6 \%$ in total F2), chloroform (32.4\% in F3), chloroform/ether (20.2\% in F4) and ethanol/chloroform (9.5\% in F7). After light exposure,

Table I. Elemental analysis of BAL 150 (before and after different durations of light exposure, as days).

\begin{tabular}{lccccc}
\hline Light exposure & $C$ & $H$ & $N$ & $O$ & $S$ \\
\hline 0 & 81.70 & 7.27 & 0.99 & 2.40 & 6.65 \\
30 days & 81.21 & 7.25 & 0.84 & 2.19 & 6.49 \\
80 days & 82.10 & 7.24 & 0.85 & 2.39 & 6.46 \\
140 days & 82.25 & 7.39 & 0.84 & 2.12 & 6.46 \\
\hline
\end{tabular}




\section{Original articles}

significant variations in gravimetric data were observed in the F2-3 subfraction (from 10.7 to $13.8 \%$ ) and in fractions eluted by methanol (2.8 to $7.5 \%$ in F6) and ethanol/chloroform (9.5 to $4.1 \%$ in F7) suggesting occurence of changes in functionnality.

\section{Fraction analyses before light exposure}

\section{Fraction 1}

Elution with hexane yielded two limpid subfractions, F1-1 and F1-2, distinguished under UV light only, F1-2 showing blue at $254 \mathrm{~nm}$. The FTIR spectrum of F1-1 was character-

Table II. Main bands in crude oil asphaltene FTIR spectra.

\begin{tabular}{ll}
\hline Wavenumbers $\left(\mathrm{cm}^{-1}\right)$ & \multicolumn{1}{c}{ Assignement } \\
\hline $3500-3100$ & $\vee \mathrm{OH}$ \\
$3100-3000$ & $\vee \mathrm{CH}$ aromatic \\
$3000-2800$ & $\vee \mathrm{CH}_{3}, \mathrm{CH}_{2}, \mathrm{CH}$ \\
1742 & $\vee \mathrm{C}=\mathrm{O}$ ester \\
1695,1650 & $\vee \mathrm{C}=\mathrm{O}$ ketone \\
$1606-1595$ & $\vee \mathrm{C}=\mathrm{C}_{\text {aromatic }}$ \\
1460 & $\delta \mathrm{CH}_{3}, \mathrm{CH}_{2}$ \\
1377 & $\delta \mathrm{CH}_{3}$ \\
1030 & $\vee \mathrm{S}=\mathrm{O}$ sulfoxyde \\
866 & $\gamma \mathrm{CH}_{\text {aromatic }}(1$ isolated $\mathrm{CH})$ \\
810 & $\gamma \mathrm{CH}_{\text {aromatic }}(2$ adjacent $\mathrm{CH})$ \\
747 & $\gamma \mathrm{CH}_{\text {aromatic }}(4$ or 5 adjacent $\mathrm{CH})$ \\
720 & $r\left(\mathrm{CH}_{2}\right)_{n}$ with $n \geq 4$ \\
\hline
\end{tabular}

Table III. Fraction to fraction gravimetric data of BAL 150 asphaltenes (before and after different durations of light exposure, as days).

\begin{tabular}{|c|c|c|c|c|c|}
\hline \multirow{2}{*}{$\begin{array}{l}\text { Elution } \\
\text { solvent }\end{array}$} & \multirow[t]{2}{*}{ fraction } & \multirow[b]{2}{*}{0} & \multicolumn{3}{|c|}{$\%$ mass after light exposure } \\
\hline & & & 30 days & 80 days & 140 days \\
\hline \multirow[t]{2}{*}{ hexane } & F1-1 & 1.5 & 0.2 & 1.9 & 1.8 \\
\hline & F1-2 & 0.8 & 0.3 & 0.8 & 0.8 \\
\hline hexane/ & F2-1 & 0.4 & 0.2 & 0.3 & 0.6 \\
\hline toluene & F2-2 & 0.5 & 0.2 & 0.6 & 0.6 \\
\hline & F2-3 & 10.7 & 13.9 & 13.5 & 13.8 \\
\hline chloroform & F3 & 32.4 & 35.7 & 36.5 & 32.0 \\
\hline $\begin{array}{l}\text { chloroform/ } \\
\text { ether }\end{array}$ & $\mathrm{F} 4$ & 20.2 & 19.0 & 19.2 & 18.8 \\
\hline ether/ethanol & F5 & 2.8 & 2.7 & 2.4 & 2.2 \\
\hline methanol & F6 & 2.8 & 3.4 & 3.5 & 7.5 \\
\hline $\begin{array}{l}\text { ethanol/ } \\
\text { chloroform }\end{array}$ & F7 & 9.5 & 4.2 & 4.3 & 4.1 \\
\hline ethanol/THF & F8 & 4.5 & 5.3 & 5.0 & 5.3 \\
\hline $\begin{array}{l}\text { ethanol/ } \\
\text { pyridine }\end{array}$ & F9 & 4.3 & 4.7 & 4.9 & 4.5 \\
\hline pyridine & F10 & 1.6 & 1.9 & 1.6 & 1.7 \\
\hline $\begin{array}{l}\text { triethylamine, } \\
\text { acetone } \\
\text { ethyl acetate }\end{array}$ & F11 & 1.0 & 1.3 & 1.0 & 2.1 \\
\hline
\end{tabular}

istic of alkanes, without evidence of aromatic rings. Absorption bands were assigned to stretching at $2955 \mathrm{~cm}^{-1}$ ( $\left.\vee \mathrm{CH}_{3}\right), 2922 \mathrm{~cm}^{-1}\left(\vee \mathrm{CH}_{2}, \mathrm{CH}_{3}\right), 2851 \mathrm{~cm}^{-1}\left(\vee \mathrm{CH}_{2}\right)$, and $2865 \mathrm{~cm}^{-1}$ as a shoulder $\left(\mathrm{V} \mathrm{CH}_{3}\right)$, to bending at $1460 \mathrm{~cm}^{-1}$ $\left(\delta \mathrm{CH}_{3}, \mathrm{CH}_{2}\right)$ and $1377 \mathrm{~cm}^{-1}\left(\delta \mathrm{s}_{3}\right)$, and to rocking at $720 \mathrm{~cm}^{-1}\left(r \mathrm{CH}_{2}\right)$ linked to $-\left(\mathrm{CH}_{2}\right) n$ groups (with $n \geq 4$ ). Gas chromatographic data confirmed that F1-1 contained alkanes only, with characteristic patterns of linear and branched molecules from $\mathrm{C}_{10}$ to $\mathrm{C}_{30}$. Note that all abovelisted IR bands ( $r \mathrm{CH}_{2}$ excepted) were observed in other fractions as well, evidencing the ubiquous importance of aliphatic groups in asphaltene structures. F1-2 displayed the same spectral characteristics as F1-1, but associated to some extra weak bands at $3100-3000 \mathrm{~cm}^{-1}$ ( $\mathrm{V} \mathrm{CH}$ ar), $1600 \mathrm{~cm}^{-1}$ $\left(\vee \mathrm{C}=\mathrm{C}\right.$ ar) and $900-700 \mathrm{~cm}^{-1}(\gamma \mathrm{CH}$ ar) assigned to aromatic rings. Alkanes $\left(\mathrm{C}_{20}-\mathrm{C}_{32}\right)$ were also detected by GC/MS. UV-fluorescence data (Tab. IV) only seemed to indicate a weak aromatic condensation at $333 \mathrm{~nm}$ (alkylbenzenes with long chains).

\section{Fraction 2}

Elution with hexane/toluene (85:15) yielded three subfractions: F2-1 (colorless), F2-2 (yellow) and F2-3 (black). The FTIR spectrum of F2-1 was characteristic of alkylated aromatics, the absence of a $720 \mathrm{~cm}^{-1}$ band suggesting a reduction in the alkyl chain lengths $(n<4)$. A relatively weak $1600 \mathrm{~cm}^{-1}$ band was observed, in addition to bands between 900 and $700 \mathrm{~cm}^{-1}$ suggesting out-of-plane aromatic hydrogen vibrations. The latter characterized aromatic ring substitutions: $747 \mathrm{~cm}^{-1}$ (presence of 4 or 5 adjacent $\mathrm{CH}$ bonds on the same ring), $810 \mathrm{~cm}^{-1}$ (2 adjacent $\mathrm{CH}$ bonds), $866 \mathrm{~cm}^{-1}$ (single $\mathrm{CH}$ bond). GC/MS data evidenced the occurence of naphthothiophene, dibenzothiophene structures, in addition to pure aromatic compounds. As shown in table V, the F2-2 FTIR spectrum differed from the F2-1 by a relative increase in the aromatic band areas $\left(1600\right.$ and $\left.900-700 \mathrm{~cm}^{-1}\right)$ and decrease in the aliphatic band areas (1460 and $\left.1377 \mathrm{~cm}^{-1}\right)$, i.e. by a reduced aliphacity and increased aromatic condensation. Similarly, comparison of UV-fluorescence spectra (Tab. IV) showed a $359 \mathrm{~nm}$ band predominance in F2-1 versus a $418 \mathrm{~nm}$ band (2-4 condensed rings) predominance

Table IV. UV Fluorescence spectra of BAL 150 asphaltene fractions (synchronous excitation emission technique, $\Delta \lambda=23 \mathrm{~nm}$ ). underlined: wavelengths of predominant bands; bold: maximal fluorescence; sh: shoulder.

\begin{tabular}{lllllllll}
\hline F1-2 & $\underline{\mathbf{3 3 3}}$ & & & & & & & \\
F2-1 & & $\underline{\mathbf{3 5 9}}$ & 380 & $\underline{415}$ & & & & \\
F2-2 & 333 & 359 & $\underline{380}$ & $\underline{\mathbf{4 1 8}}$ & & & & \\
F2-3 & & & 380 & $\underline{418}$ & $\underline{\mathbf{4 3 0}}$ & 453 & & \\
F3 & & & 385 & 420 & 438 & $\underline{\mathbf{4 5 7}}$ & & \\
F4 & & & 385 & $\underline{420}$ & $\underline{\mathbf{4 3 3}}$ & 453 & & \\
F5 & & & 385 & $\underline{\mathbf{4 1 8}}$ & sh & 455 & & \\
F6 & 361 & 380 & $\underline{415}$ & sh & $\underline{455}$ & 485 & \\
F7 & & & & 439 & 469 & $\underline{\mathbf{4 9 0}}$ & 513 \\
F8 & & & 421 & & 463 & $\underline{\mathbf{4 9 5}}$ & 513 \\
F9 & & & & & 430 & $\underline{\mathbf{4 6 9}}$ & 493 & 513 \\
F10 & & & & & 436 & 479 & $\underline{\mathbf{4 9 8}}$ & 514 \\
F11 & & 385 & $\underline{\mathbf{4 1 5}}$ & & 453 & & \\
\hline
\end{tabular}


Table V. FTIR aliphaticity and aromaticity indices of fractions 2 of BAL 150 asphaltenes, (before light exposure).

\begin{tabular}{llcc}
\hline index & area ratio & $F 2-1$ & $F 2-2$ \\
\hline aromaticity & $1600 \mathrm{~cm}^{-1}$ band area/total area & 0.02 & 0.04 \\
aliphaticity & $\begin{array}{l}1460+1377 \mathrm{~cm}^{-1} \text { band areas/ } \\
\text { total area }\end{array}$ & 0.26 & 0.17 \\
aromaticity & $\begin{array}{l}900-700 \mathrm{~cm}^{-1} \text { band area/ } \\
\text { total area }\end{array}$ & 0.26 & 0.31 \\
\hline
\end{tabular}

Total area $=$ area between 1800 and $650 \mathrm{~cm}^{-1}$.

in F2-2. Note that the $1030 \mathrm{~cm}^{-1}$ band observed in the F22 FTIR spectrum is generally attributed to sulfoxyde groups [31]. FTIR patterns of F2-1 and F2-2 were rather similar between 900 and $700 \mathrm{~cm}^{-1}$, with predominance of the $810 \mathrm{~cm}^{-1}$ band (2 adjacent $\mathrm{C}-\mathrm{H}$ bonds). Finally, the F2-3 FTIR spectrum did not differ much, besides the emergence of a $1695 \mathrm{~cm}^{-1}$ band (Fig. 1), assigned to carbonyl groups conjugated with aromatic rings, like in alkylarylketone and naphthenoarylketone. As opposed to F2-1 and F2-2, F2-3 showed predominance in the $866 \mathrm{~cm}^{-1}$ band (single $\mathrm{C}-\mathrm{H}$ bond) versus the $810 \mathrm{~cm}^{-1}$ band ( 2 adjacent $\mathrm{CH}$ bonds), i.e. more substitution of the aromatic rings. Comparison of UVfluorescence spectra showed the disappearance of the $359 \mathrm{~nm}$ band in F2-3, and (besides two maxima at 380 and at $418 \mathrm{~nm}$ ) the emergence of two additional bands: a predominant one at $430 \mathrm{~nm}$ and another one at $453 \mathrm{~nm}$ (4 linearly condensed rings).

\section{Fraction 3}

F3, eluted with chloroform, collected the most abundant asphaltene fraction (32.4\%). FTIR spectra displayed by F3 and by F2-3 were remarkably close (Fig. 1), but a relative increase in the $1600 \mathrm{~cm}^{-1}$ band and a relative reduction in absorbance between 900 and $700 \mathrm{~cm}^{-1}$ indicated more aromatic ring condensation in $\mathrm{F} 3$ than in F2-3. This trend was confirmed by UV-fluorescence, that showed a definite predominance of the $457 \mathrm{~nm}$ versus the $430-438 \mathrm{~nm}$ band, i.e. an increased aromatic condensation and/or presence of heterocyclic and naphthenic species. The F3 ${ }^{1} \mathrm{H}$ NMR spec- trum showed $\mathrm{a} \approx 1.7 \mathrm{ppm}$ band assigned to naphthenic structures $[17,24,25]$, plus an enhanced resonance in the broad 2 - 3.4 ppm band ( $\mathrm{H} \alpha$ to aromatic rings), both features consistent with the presence of naphthenoaromatic structures. The F3 FTIR spectrum (Fig. 1) displayed moderate absorption bands between 3400 and $3200 \mathrm{~cm}^{-1}$ indicating the presence of hydroxyl groups.

\section{Fraction 4}

F4, eluted with chloroform/diethylether (90:10), showed an aromatic condensation admittedly similar or slightly weaker than F3, with a predominance of the 420 and $433 \mathrm{~nm}$ bands in the UV fluorescence spectrum, as described for F2-3. Figure 1 compares FTIR spectra of F2-3, F3 and F4. The latter clearly indicated an increase in carbonyl groups, with an absorbance ratio at $1695 \mathrm{~cm}^{-1}(\mathrm{C}=\mathrm{O})$ and at $1600 \mathrm{~cm}^{-1}$ culminating at 0.83 versus 0.59 for $\mathrm{F} 3$. Absorption between 3400 to $3100 \mathrm{~cm}^{-1}$ was also enhanced with reference to $\mathrm{F} 3$, and here centered at $3250 \mathrm{~cm}^{-1}$. The shoulder at $1650 \mathrm{~cm}^{-1}$ should be assigned to carbonyl groups more conjugated than the carbonyl groups responsible for the $1695 \mathrm{~cm}^{-1}$ band, that corresponded to diarylketone structures. Finally, increases in absorbance and band width were also observed at $1030 \mathrm{~cm}^{-1}$.

\section{Fraction 5}

The FTIR spectrum of F5, eluted with diethylether/ethanol (97:3), was characterized by an intense absorption band between $3300-3200 \mathrm{~cm}^{-1}$ centered at $3258 \mathrm{~cm}^{-1}$, another strong band at $1200 \mathrm{~cm}^{-1}$ (sometimes split into 1203 and $1188 \mathrm{~cm}^{-1}$ ), and by an increase in absorbance of the $1600 \mathrm{~cm}^{-1}$ band. These features were consistent with the presence of phenolic groups, the first band corresponding to the phenol $\mathrm{OH}$ stretching vibrations. In phenols, the $\mathrm{O}-\mathrm{H}$ bending and $\mathrm{C}-\mathrm{O}$ stretching vibrations are strongly coupled and give rise to a marked band between 1260 and $1180 \mathrm{~cm}^{-1}$ [15]. On the other hand, the $1600 \mathrm{~cm}^{-1}$ band has now been assigned to aromatic $\mathrm{C}=\mathrm{C}$ bond stretching vibrations, while its intensity depends on the nature of the ring substituents [19]. With OH groups (mainly in ortho and para positions) absorption can be especially intense. Summarily, a weak $1600 \mathrm{~cm}^{-1}$ band stands for non-polar aromatic compounds, while a strong one stands for polar aromatic compounds, such as phenols. Absorption at $1650-1660 \mathrm{~cm}^{-1}$ and
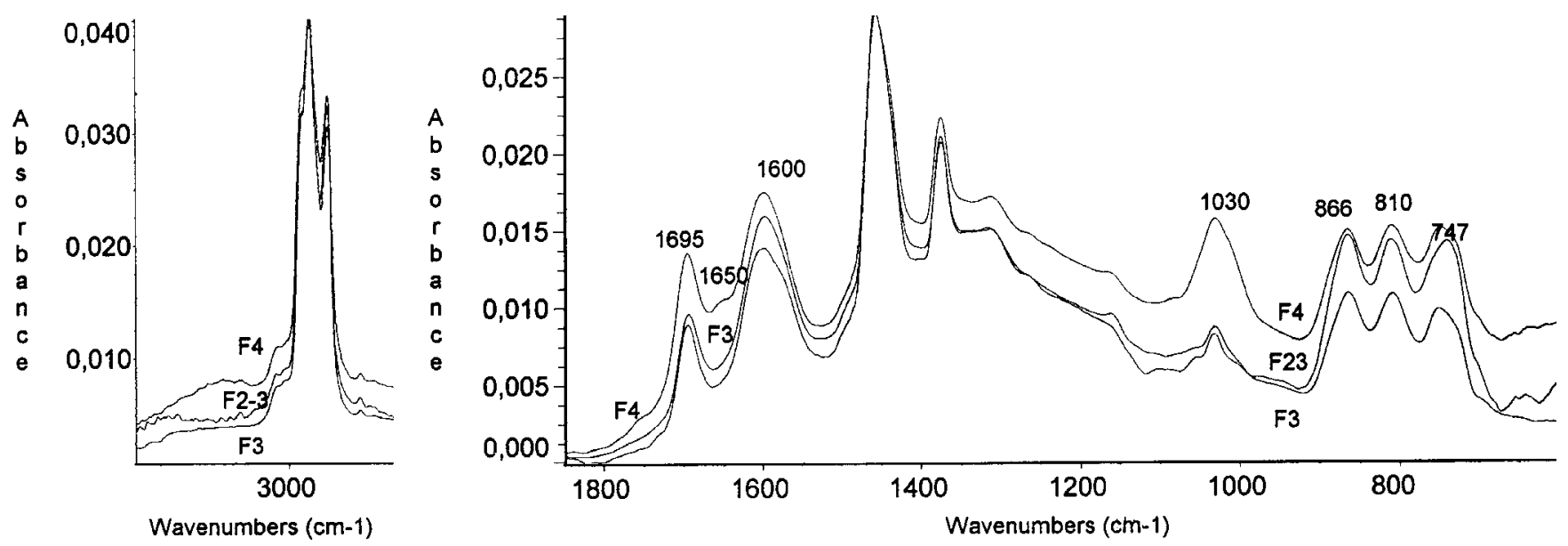

Fig. 1. FTIR spectra of fractions F2-3, F3 and F4 of BAL 150 asphaltenes before light exposure. 


\section{Original articles}

$1695 \mathrm{~cm}^{-1}$ were observed in F5 like in F4, except that the $1660 \mathrm{~cm}^{-1}$ band (diarylketone) was here predominant. UV fluorescence data are described in the next paragraph.

\section{Fraction 6}

F6, eluted with methanol, displayed the same IR spectral characteristics as F5 (intense bands at $3250 \mathrm{~cm}^{-1}, 1600 \mathrm{~cm}^{-1}$, and $1200 \mathrm{~cm}^{-1}$ showing the presence of phenols), but a marked decrease in the intensity of the $1660 \mathrm{~cm}^{-1}$ band. Both F5 and F6 UV-fluorescence spectra showed six maxima between 360 and $490 \mathrm{~nm}$ ( 2 to 5 rings and more), indicating that those two fractions contained all the phenol and polyphenol structures, irrespective of the number of rings in their hydrocarbon skeletons.

\section{Fraction 7}

The FTIR spectrum of F7, eluted with ethanol/chloroform (3:97), displayed bands between 1300 and $1000 \mathrm{~cm}^{-1}$, due to occurrence of ether bonds, and assigned to $\mathrm{R}-\mathrm{O}-\mathrm{R}$ structures $\left(1122\right.$ and $\left.1069 \mathrm{~cm}^{-1}\right)$, to the $\mathrm{O}-\mathrm{C}\left(\mathrm{sp}^{3}\right)$ bond of $\mathrm{Ar}-$ $\mathrm{O}-\mathrm{R}$ structures $\left(1034 \mathrm{~cm}^{-1}\right)$, and to $\mathrm{O}-\mathrm{C}\left(\mathrm{sp}^{2}\right)$ bonds of $\mathrm{Ar}-$ $\mathrm{O}-\mathrm{R}$ or $\mathrm{Ar}-\mathrm{O}-\mathrm{Ar}$ structures $\left(1265 \mathrm{~cm}^{-1}\right)$ [16]. Several UV-fluorescence maxima $(439,469,490$ and $513 \mathrm{~nm})$ were observed with a predominant band at $490 \mathrm{~nm}$, evidencing a greater aromatic condensation ( 5 condensed rings or more) in F7 compared to the six first eluted fractions.

\section{Fractions 8, 9, 10}

These 3 fractions were respectively eluted with ethanol/tetrahydrofuran (3:97), ethanol/pyridine (3:97) and pyridine. Using deconvolution routines (Fig. 2), two bands were detected in the FTIR spectrum of F8, at 1726 and $1773 \mathrm{~cm}^{-1}$, and assigned to $\mathrm{Ar}-\mathrm{CO}-\mathrm{O}-\mathrm{R}$ and $\mathrm{R}-\mathrm{CO}-\mathrm{O}-\mathrm{Ar}$ ester structures respectively [16]. These bands, relatively weak in the case of BAL 150, were intense in the case of fractions 8 extracted from either PBS or Irak crude oil asphaltenes. Deconvolution revealed another band at $1715 \mathrm{~cm}^{-1}$ associated with a broad band $\left(3500-2200 \mathrm{~cm}^{-1)}\right.$ characteristic of carboxylic groups. This band also was much more intense in PBS asphaltenes than in BAL 150, and its coincidence with the $v \mathrm{COOH}$ band rendered an assignement to carboxylic $\vee \mathrm{C}=\mathrm{O}$ especially evident. UV fluorescence data of F8, F9 and F10 were similar to above described, with

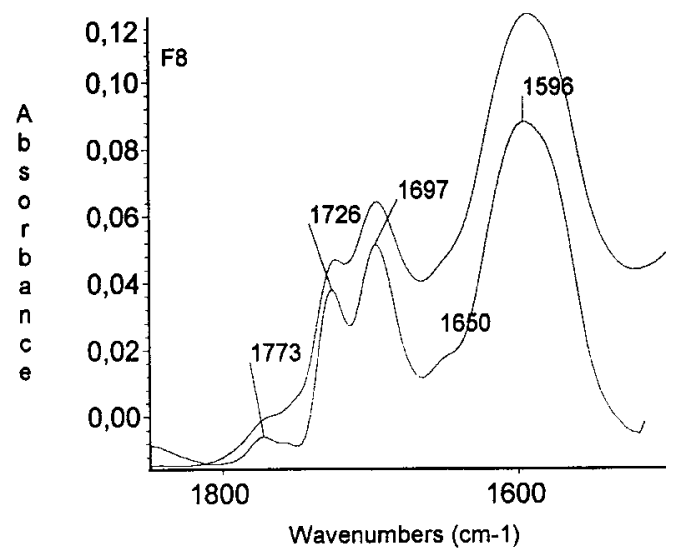

Fig. 2. FTIR spectrum of fraction F8 of BAL asphaltenes before light exposure: before (top) and after deconvolution (bottom). bands at 463-469, 490-495 (maximal intensity) and $513 \mathrm{~nm}$.

\section{Fraction 11}

The FTIR of F11, eluted with triethylamine, acetone and ethylacetate, evidenced slighlty aromatic compounds, with poorly resolved bands between 1800 and $1500 \mathrm{~cm}^{-1}$ more suggestive of highly polar structures - most probably residual resins. Deconvolution revealed bands at 1772, 1716, 1664,1606 and $1564 \mathrm{~cm}^{-1}$. UV fluorescence data confirmed the low aromaticity of this fraction, with a maximum at $415 \mathrm{~nm}$ ( $\leq 4$ condensed rings).

\section{Fraction analyses after light exposure}

\section{Fractions 1 and 2}

After 30 days of light exposure, F1 and F2-1 were partly oxidized. Aliphatic ketones appeared in F2-2, as a result of photo-oxidation of alkanes and alkyl chains. After 80 and 140 days, "new" alkanes and long-chained alkyl benzenes were produced. They might either originate from radical reactions, or represent compounds originally trapped in the asphaltene matrix [30] and released by its photo-oxidation [10].

As shown in table III, the gravimetric percentage of F23 increased from $10.7 \%$ before to $13.8 \%$ after light exposure. New arylalkylketones, resulting from oxidation of alkylaromatic structures, appeared in F2-3, but without change in the ratio $\vee \mathrm{C}=\mathrm{O} / \mathrm{V} \mathrm{C}=\mathrm{C}$ ( Tab. $\mathrm{VI}$ ), i.e. without addition of new carbonyl groups to the pre-existent arylalkylketones (and naphtheno arylketones).

\section{Fraction 3}

After light exposure, the F3 FTIR spectrum basically showed an increase in the $1695 \mathrm{~cm}^{-1}$ band (Tab. VI and Fig. 3), while ${ }^{1} \mathrm{H}$ NMR data evidenced marked decreases in the 1.7 and 2-3.4 ppm peaks assigned to naphthenic structures with $\mathrm{CH}_{2}$ in $\alpha$ of aromatic rings. No significant changes were observed in the $\mathrm{vOH}$ absorption band of IR spectra.

\section{Fraction 4}

The $v \mathrm{C}=\mathrm{O} / v \mathrm{C}=\mathrm{C}$ ratio remained constant throughout the light exposure (Tab. VI), but the $1650 \mathrm{~cm}^{-1}$ band underwent stringent changes. After 30 days, an increase in absorbance was first observed (Fig. 3); after 80 days, deconvolution evidenced a splitting of this band. An increase in the "sulfoxyde" $1030 \mathrm{~cm}^{-1}$ band also was simultaneously observed.

Table VI. FTIR oxidation indices of fractions $2-3,3$ and 4 of BAL 150 asphaltenes, (before and after different durations of light exposure, as days).

\begin{tabular}{lcccc}
\hline Fraction & \multicolumn{4}{c}{$\begin{array}{c}\text { Absorbance ratio } \\
(v \mathrm{C}=\mathrm{O} / \mathrm{v} \mathrm{Cm}=\mathrm{C})\end{array}$} \\
& 0 & 30 days & 80 days & 140 days \\
\hline F2-3 & 0.62 & 0.63 & 0.60 & 0.59 \\
F3 & 0.55 & 0.67 & 0.65 & 0.65 \\
F4 & 0.83 & 0.83 & 0.82 & 0.82 \\
\hline
\end{tabular}



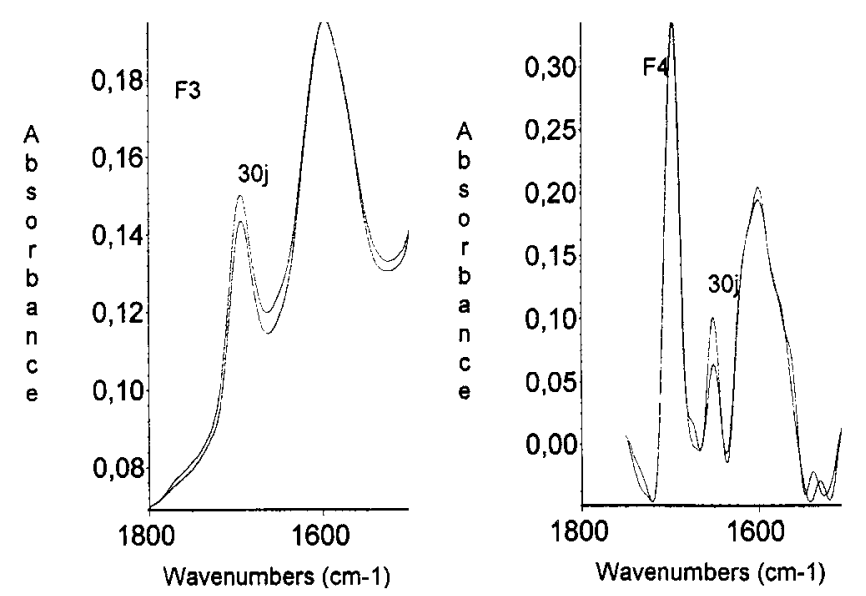

Fig. 3. FTIR spectra (after deconvolution) of fractions F3 and F4 of BAL 150 asphaltenes before (bottom) and after 30 days of light exposure (top).

\section{Fractions 5 and 6}

In these basically phenolic fractions, light exposure induced a further enrichment in phenol compounds. Figure 4 compares F5 FTIR spectra before and after light exposures during 30,80 and 140 days, showing the increase in absorbance at $3500-3200,1600,1200$, and $1043 \mathrm{~cm}^{-1}$. The band that culminated between 3500 and $3200 \mathrm{~cm}^{-1}$ in the spectra obtained from solid preparations was further investigated after dissolution in $\mathrm{CCl}_{4}$, and showed splitted (3435 and $3260 \mathrm{~cm}^{-1}$ ) under liquid state. This conjunction of bands at 3435 and $1043 \mathrm{~cm}^{-1}$ (respectively assigned to $\mathrm{v} \mathrm{OH}$ and to $v \mathrm{CO})$ suggested that alcohols were formed during light exposure.

\section{Fraction 7 to 11}

After light exposure, no oxidized species were detected anew other than those existing before: arylalkylketones, diarylketones, sulfoxydes, phenols, ethers, esters and carboxylic acids. In fraction 11, a remarkable increase in absorbance was observed between 1800 and $1500 \mathrm{~cm}^{-1}$.

\section{Summary}

\section{Global approaches}

Analyses without fractionation provided little information about the effects of light exposure on isolated asphaltenes. In several studies however, this kind of approach had been successfully used for addressing structural changes in fractions rich in aromatic compounds.

For instance, using UV-fluorescence spectroscopy to study polyaromatic hydrocarbons, Mille et al. [32] and Baudot et al. [33] demonstrated that emission wavelengths obviously depended on the number and position of condensed aromatic rings. Kister et al. [34] further observed that addition of alkyl chains, naphthenic rings, and sulphur heterocycles to the basic polyaromatic skeleton resulted in bathochromic effects. Moreover, structural indexes, computed from area ratios of characteristic IR bands, enabled other authors to assess several characteristics (aromaticity, ramification, condensation, substitution) of liquid coals [34] or of bitumens [11]. Under our experimental conditions, even after 140 days of light exposure, none of these indices enabled us to detect any significant structural change in hydrocarbon skeleton [21].

\section{Fraction to fraction analyses}

Sequential elution solvent chromatography was anew proven a good tool to fractionate asphaltenes according to chemical functionality. This technique was further improved: a) by partitioning the two initial fractions (eluted as usually [1417] by hexane and toluene) into five subfractions of different contents: alkanes, long-chain alkylbenzenes, alkylated polyaromatics (or naphtheno-polyaromatics), and oxidized polyaromatics (alkylarylketones or naphthenoarylketones); and b) by completing elution with three additional solvents (triethylamine, acetone and ethylacetate) to extract residual compounds trapped in the asphaltenic matrix. Subsequent analyses allowed us to monitor functional changes fraction to fraction. Most alkanes and alkylbenzenes were photo-oxidized mainly into alkylketones after 30 days. Pure aromatic compounds were simultaneously oxidized, as shown by an increase in arylketones (from 10.7 to almost 14\%). In the predominant 3 to 4-ring naphthenoaromatic fraction (containing ketonic, hydroxyl and sulfoxyde groups), light
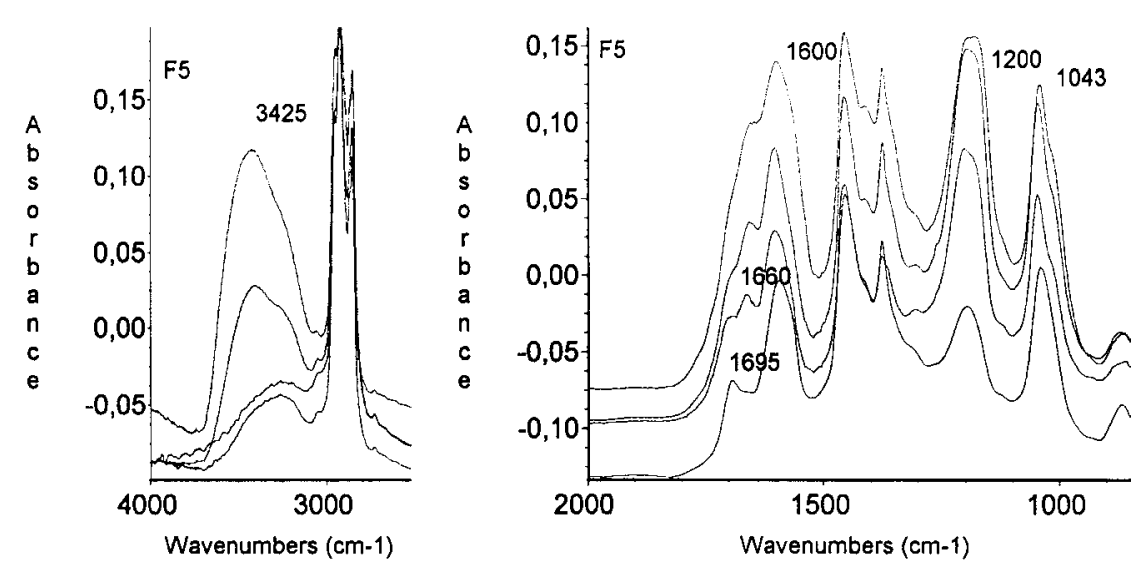

Fig. 4. FTIR spectra of fraction F5 of BAL 150 asphaltenes: (from bottom to top) before and after 30, 80 and 140 days of light exposure. 


\section{Original articles}

exposure induced qualitative changes, especially an increase in carbonyl groups by oxidation of a carbon in aromatic rings, as suggested by ${ }^{1} \mathrm{H}$ NMR data. The next-eluted fraction, also containing mainly naphthenoaromatic compounds, but more carbonyl, hydroxyl and sulfoxyde groups, plus diarylketone structures, showed an increase in sulfoxyde groups and diarylketones. The 2 to 5-ring phenolic fractions underwent important ponderal changes, with an increase in phenol groups (polyphenols) and likely formation of alcohols. The more polar, next-coming fractions (5 and more condensed rings) showed the same chemical functions (alkylarylketone, diarylketone, sulfoxyde, ether, ester, carboxylic acid) with little evolution throughout light exposure. The compounds separated by additionnal elutions also showed some increase in functional groups.

\section{Conclusion}

Previous works [5,10,35] showed that crude oils and petroleum residues are considerably influenced by irradiation changing its physico-chemical characteristics (solubilization, aggregation, emulsification properties).

In this work, which concerns light exposure of only the asphaltenic fraction of BAL 150 crude oil, separated from aliphatics, aromatics and resins, we have observed moderate structural changes, basically limited to increases in functional groups (phenols, ketones, sulfoxydes, carboxylic groups and possibly alcohols), and little variation if any in the hydrocarbon skeletons of the compounds that constituted the asphaltene matrix or were trapped in. These structural changes are not detectable by global analyses as shown by elemental analysis data which do not exhibit significant variations. Particularly elemental analyses do not indicate an increase of oxygen content while the main conclusions from fraction to fraction analysis is an oxidation of asphaltenes.

It would be interesting to know if these moderate structural changes are sufficient to induce large physico-chemical modifications of asphaltenes. This could arise from a superficial oxidation, however deeply altering the hydrophilic character of polycyclic sheets. We have observed that alkanes and long chain alkyl benzenes, trapped in the asphaltenic matrix, were released during light exposure. These hydrocarbons could be photooxidized to give tensioactive acid compounds. This problem could be investigated in depth in the future by the determinations of overall physical indices (viscosity, rheological parameters, average molecular weights...) and by the methods of colloidal chemistry.

\section{References}

1. Payne, J. R.; Phillips, C. R. Env. Sci. Technol. 1985, 19, 569579.

2. Jordan, R. E. Doctor Thesis, University of California San Diego and San Diego State University, 1991.
3. Bobra, M. Research Report, River Road Environmental Centre, Ottawa, Canada, 1992.

4. El Anba-Lurot, F. Photodégradation des hydrocarbures et des organoétains en milieu marin. Identification des photoproduits et mécanismes, Thèse de Doctorat en Sciences, Université d'Aix-Marseille, 1995.

5. Nicodem, D. E.; Fernandes, M. C. Z.; Guedes, C. L. B.; Correa, R. J. Biogeochem. 1997, 39, 121-138.

6. Rontani, J. F Trends Photochem. Photobiol. 1997, 4, 125-136.

7. Martin, K. G.; Symposium on the Science of Asphalt in Construction, ACS, 1971.

8. Mill, T.; Tse, D. Abstr. Pap. Am. Chem. Soc. 1990, $200,44$.

9. Tjessem, K.; Kobberstad, O.; Aaberg, A. Chemosphere 1983, 12, 1395-1406.

10. Tjessem, K.; Aaberg, A. Chemosphere 1983, 12, 1373-1394.

11. Pieri, N.; Planche, J. P.; Kister, J. Analusis 1996, 24, 113-122.

12. Yen, T. F. Preprints Div. Petrol. Chem. ACS, 1972, 17, F102114.

13. Speight, J. G. Preprints Div. Petrol. Chem. ACS, 1989, 34, 321-328.

14. Farcassiu, M. Fuel 1977, 56, 9-14.

15. Seshadri, K. S.; Cronauer, D. C. Fuel 1983, 62, 1436-1444.

16. Seshadri, K. S.; Young, D. C.; Cronauer, D. C. Fuel 1985, 64, 22-28.

17. Jacobs, F. S.; Filby, R. H. Fuel 1983, 62, 1186-1192.

18. AFNOR AFNOR-Prod. Pétrol. 1982, NF T 60-15, 1-6.

19. Guiliano, M.; Mille, G.; Kister, J.; Muller, J. F. J. Chim. Phys. 1988, 85, 963-970.

20. Yen, T. F.; Wu, W. H., Chilingar, G. V. Energy Sources 1984, 7, 203-235.

21. Boukir, A.; Guiliano, M.; Doumenq, P.; El Hallaoui, A.; Mille, G. C. R. Acad. Sci. Paris, 1.1, Ser. IIa, 1998, 597-602.

22. Yen, T. F.; Wu, W. H.; Chilingar, G. V. Energy Sources 1984, 7, 275-304.

23. Speight, J. G. Fuel 1970, 49, 134-145.

24. Favre, A.; Boulet, R. Rev. Inst. Franc. Pétrole 1984, 39, 485496.

25. Dahbi, A.; Bitar, H.; Bennouma, C.; Béguin, C. G.; Faure, R. Analusis 1992, 20, 239-243.

26. Kotlyar, L. S.; Morat, C.; Ripmeester, J. A. Fuel 1991, 70, 90-94.

27. Shu-An-Qiam, Peng-Zhou, Zhang; Bai-Ling, Li Fuel 1985, 64, 1085-1091.

28. Seshadri, K. S.; Albaugh, E. W.; Bacha, J. D. Fuel 1982, 61, 336-340.

29. Gillet, S.; Rubini, P.; Delpuech, J. J.; Escalier, J. C.; Valentin, P. Fuel 1981, 60, 221-225.

30. Myhr, M. B.; Schou, L.; Skjetne, T.; Krane, J. Org. Geochem. 1990, 16, 931-941.

31. Petersen, J. C. Transport Res. Record 1986, 1096, 1-11.

32. Mille, G.; Guiliano, M.; Kister, J. Org. Geochem. 1988, 13, 947-952.

33. Baudot, P.; Viriot, M. L.; André, J. C.; Jezequel, J. Y.; Lafontaine, M. Analusis 1991, 19, 85-97.

34. Kister, J.; Pieri, N.; Alvarez, R.; Diez, M. A.; Pis, J. J. Energy Fuels 1996, 10, 948-957.

35. Lamathe, J. C. R. Acad. Sci. Paris, Ser. II 1982, 295, 301-302. 